\title{
THE X-RAY PROPERTIES OF HIGH REDSHIFT QUASI-STELLAR OBJECTS
}

\author{
Scott F. Anderson ${ }^{1,2}$ and Bruce Margon ${ }^{1}$ \\ Department of Astronomy, FM-20 \\ University of Washington \\ Seattle, Washington 98195, USA
}

\begin{abstract}
We describe a program aimed at characterizing the X-ray emission of high redshift QSOs. We have obtained slitless spectra of 50 high galactic latitude fields previously imaged at very high levels of sensitivity by the Einstein Observatory, generally for original goals unrelated to QSOs. Our survey, covering $\sim 17 \mathrm{deg}^{2}$ of sky to limiting magnitude $B_{\text {cont }} \sim 21$, has yielded $\sim 400$ previously uncatalogued QSO candidates, each with sensitive new X-ray information available. About 100 of these objects, constituting a "high confidence" set of QSOs, chiefly in the redshift range $1.7<z<3$ and thus complementary to previous samples with X-ray data, are used to derive the X-ray properties of high redshift QSOs. Even at these most sensitive available X-ray flux levels, only about $25 \%$ of the objects are positively detected in X-rays; thus extensive attention has been given to proper treatment of the upper-limit information. We find a mean optical-to-X-ray slope parameter for the sample of $\alpha_{o x}^{\text {eff }}=1.50$. Our results are combined with those of previous surveys to estimate the fraction of the diffuse X-ray background radiation due to QSOs. QSOs are capable of supplying the majority of the radiation, but the chief contribution comes from an annulus of intermediate redshift, moderate luminosity objects.
\end{abstract}

\section{INTRODUCTION}

X-ray observations from the Einstein Observatory have increased tremendously our understanding of the X-ray properties of quasi-stellar objects (QSOs), and permitted for the first time reasonably quantitative estimates of the contributions of QSOs to the diffuse Xray background radiation. Nonetheless controversy remains on a variety of crucial points, e.g., if and how the X-ray properties evolve with redshift and luminosity, and whether QSOs in fact are the dominant contributor to the background. Part of the current uncertainties is doubtless due to the intrinsic complexity of the problem. QSOs exhibit a wide dispersion in their optical and radio properties, so there is little reason to hope that the $\mathrm{X}$-ray emission will be simpler. However, an additional complexity is introduced by the necessary but peculiar selection biases of past X-ray observations of QSOs.

1 Visiting Astronomer, Kitt Peak National Observatory, and Cerro Tololo Interamerican Observatory, National Optical Astronomy Observatories, operated by AURA Inc., under contract with the NSF.

2 Current address: Steward Observatory, University of Arizona, Tucson, AZ 85721, USA. 
Most previous studies have followed one of three approaches. (a). QSOs catalogued previous to the X-ray observations have been studied intensively (e.g., Zamorani et al. 1981). These "famous" objects are often atypical of the QSO population, however, as they tend to have abnormally high radio or optical luminosity when selected by these traits. Further, these studies have revealed a remarkably strong correlation between QSO $\mathrm{X}$-ray and radio luminosity, making extrapolations of the rare but well-observed radio objects to the more common but radio-quiet population rather dangerous. Finally, Elvis and Lawrence (1985) have recently demonstrated that radio and optically selected QSOs have very different $\mathrm{X}$-ray spectral slopes, thus calling into question the $\mathrm{X}$-ray intensity calibration of a large fraction of previously published work. (b). X-ray selected QSOs, found as the optical counterparts of serendipitously detected X-ray sources, have been studied by several groups, with particularly large samples available in Gioia et al. (1984) and Margon et al. (1985). At the risk of stating the obvious, we note that $\mathrm{X}$-ray selected objects must by hypothesis be $\mathrm{X}$-ray bright, and such lists are thus badly biased against QSOs with a small ratio of X-ray to optical luminosity; a population of "X-ray quiet" QSOs would have escaped detection entirely. The very skewed redshift distribution of such X-ray selected samples, which prove to have $\left\langle z>\sim 0.4\right.$ even at $B_{\text {lim }}=20$, also stands as a warning against simple application of the $\mathrm{X}$-ray properties of this sample to the entire QSO population. (c). The Braccesi sample of UV-excess selected QSOs has been observed at high sensitivity at X-ray wavelengths by Marshall et al. (1984). While this sample is chosen without advance bias towards its $\mathrm{X}$-ray properties, there are other potential complications. The sample size is small, and the nature and completeness of the Braccesi sample has been somewhat controversial (Veron 1983). By selection, the sample has a modest mean redshift of $\langle z\rangle \sim 1$.

\section{OBSERVATIONS}

We have recently completed a program aimed at complementing these previous studies through use of an orthogonal selection technique for QSOs with X-ray information. The data base accumulated by the Einstein Observatory of course contains information on thousands of still anonymous but reasonably bright QSOs. We know from past studies of the surface density of QSOs, for example, that each $0.5 \mathrm{deg}^{2}$ image obtained by Einstein included coverage of about 10 QSOs at $V<19.5$, regardless of the original purpose of that image; this extensive, already extant QSO data base can be exploited if these currently uncatalogued QSOs can be discovered. We have concentrated on $50 \mathrm{X}$-ray fields obtained with the Imaging Proportional Counter (IPC) at high galactic latitude, $|b|>30^{\circ}$, which represent amongst the longest, and thus most sensitive, of all Einstein integrations. The original goals of these exposures spanned a large variety astrophysical topics, normally quite unrelated to QSOs. Typical exposure times of our fields were $(1-5) \times 10^{4} \mathrm{sec}$, and the data are thus quite competitive in sensitivity with the so-called "Deep Surveys" (Giacconi et al. 1979).

At the magnitudes conceivably relevant to these $\mathrm{X}$-ray fluxes, past experience has shown that slitless spectroscopy is a relatively complete and rapid technique for discovery of large numbers of QSOs in a restricted region of sky. Luckily the $38 \times 38$ ' area 'inside the ribs" of the IPC is very well-matched to the prime focus field of several large telescopes. For our observations we have used a grism at the prime focus of the Kitt Peak and Cerro Tololo 4-meter telescopes, and the "grens" at the Canada-France-Hawaii Telescope prime focus. Typical spectral dispersions employed were $1500 \AA \mathrm{mm}^{-1}$, which is quite sufficient 
for identification of strong emission line QSOs. The large area of the field, about 100 $\mathrm{cm}^{2}$, dictates that photographic plates must be used as detectors. For all of our fields we have obtained one or more III-aF emulsions. These plates, baked in forming gas and exposed unfiltered for about 50 minutes, reach $B_{\text {cont }} \sim 21.0$ in good seeing, and cover the $\lambda \lambda 3200-6900 \AA$ band. We chose the $\mathrm{F}$ in preference to the $\mathrm{J}$ emulsion in an attempt to gain wider wavelength coverage, at the expense of the well-known nonuniformity of the emulsion. For many of the fields we further extended the coverage by also obtaining a IV-N plate, hypersensitized with $\mathrm{AgNO}_{3}$. These plates, when exposed for $90 \mathrm{~min}$ through a Wr29 filter, can reach about $V_{\text {cont }} \sim 21.0$ in good seeing, and cover the $\lambda \lambda 6100-8900$ $\AA$ band. The total survey covered about $17 \mathrm{deg}^{2}$ on about 100 plates. With the goal of avoiding lengthy software preparation, this large but still manageable quantity of spectra (about $10^{3}$ spectra per plate) was reduced manually, with each plate scanned on several independent occasions with a binocular microscope, and underlying grid to systematize the search. A comprehensive description of the observations and reductions is given by Anderson (1985).

Our search resulted in a group of $\sim 400$ new QSO candidates, chiefly with $17.5<$ $B<21.0$, and $1.7<z<3$, although the efforts at extended wavelength coverage did result in a significant number of low redshift objects as well. By construction of the sample, each of these objects has available one of the most sensitive possible Einstein observations. Without slit spectra to confirm each object, a prohibitive task for this large, faint sample, one must of course worry about sample completeness and contamination. We have extracted from these 400 objects a subgroup of 102 "high confidence" QSOs, objects whose spectra show very prominent and/or multiple emission lines, and often exceptionally blue continua as well. We are confident that the large majority of these objects are in fact bonafide QSOs, and the remainder of the analysis described here will be confined to this sample only. In the small number of objects from this group that we have managed thus far to observe with high spectral resolution, every one has proven to be a QSO.

\section{ANALYSIS AND CONCLUSIONS}

For ease of analysis we have divided the high confidence sample into two further groups. Our "complete sample" consists of all high confidence objects with $B<19.5$ and $1.8<z<$ 3.0. Several previous studies have shown that grism selected QSOs are largely $(>80 \%)$ complete in this regime. The 40 QSOs in this group, extracted from $12.9 \mathrm{deg}^{2}$, yield an internal space density of $3.1 \mathrm{deg}^{-2}$, which is comparable to that found by previous workers. This complete sample is useful for direct estimates of the contribution of QSOs to the diffuse X-ray background radiation, free of assumptions and/or extrapolations on $\mathrm{X}$-ray evolution, or even external space density estimates. A second useful subset is hereafter referred to as our "narrow sample." This consists of 80 objects in the narrow slice of parameter space confined to $1.8<z<2.5$ and $31<\log l_{2500 \AA}<32$. This restricted sample is useful for testing and extrapolating previous predictions concerning the evolution of $\mathrm{X}$-ray properties with optical luminosity and/or redshift; this group can be treated as an empirical data point, without fear of variation of properties within the sample.

Analysis of the X-ray data at each of the coordinates of our newly discovered QSOs was performed at the Harvard-Smithsonian Center for Astrophysics. We find that despite the high level of sensitivity of the X-ray exposures, only $\sim 25 \%$ of our objects yield positive $\mathrm{X}$-ray detections. Proper treatment of the $\mathrm{X}$-ray upper limits is thus essential to maximize 
extraction of information. We have employed "survival statistics", recently discussed in an astronomical context by Feigelson and Nelson (1985) and Schmitt (1985); the socalled "detection and bounds" method of Avni et al. (1980) represents an independent rediscovery of this previously published, more general technique. We have also employed the digital image stacking technique used by Caillault and Helfand (1985) in a slightly different context, and derive equivalent results to the survival analysis. We follow past workers in the field by characterizing our results in terms of the parameter $\alpha_{o x}$, the slope of a hypothetical power law joining the $2500 \AA$ and X-ray fluxes of each QSO (Tananbaum et al. 1979), and also use the mean values, $\alpha_{o x}^{\text {eff }}$, to discuss ensemble properties. The necessary optical photometry has been obtained from image diameter measurements on the Sky Survey, and a careful calibration (Anderson 1985) convinces us that our rms uncertainties do not exceed a few tenths of a magnitude for $B<20.2$ (excluding, of course, time variability of the QSO).

We find that the fractional contribution of our "complete" sample, that is, QSOs with $B<19.5$ and $1.8<z<3.0$, to the diffuse X-ray background cannot exceed $3 \%$ of the observed background intensity. This is a direct measurement, requiring no extrapolation of possible $\alpha_{o x}$ functional dependences, for example. We can combine this result with complementary work by Marshall et al. (1984) on the "Braccesi faint" sample, which covers the range $0<z<2.2, B<19.8$. For $z>3.0$, the space density of QSOs is quite uncertain, but can very conservatively be shown to be a small fraction of all QSOs with $B<19.5$. We thus conclude that the total contribution of all QSOs with $B<19.5$ is less than $20 \%$ of the diffuse $X$-ray background.

Survival analysis of our "narrow" sample yields an ensemble value of $\alpha_{o x}^{\text {eff }}=1.50 \pm 0.03$. This illustrates that we have succeeded in probing a significantly more "X-ray quiet" class than past work; the value for the X-ray selected sample of Margon et al. (1985), for example, is $1.26 \pm 0.03$, and $90 \%$ of our objects prove to have smaller ratios of X-ray to optical luminosity than the $\alpha_{o x}^{\text {eff }}=1.42$ inferred by Marshall et al. (1984) for their UVexcess selected sample. (There is no conflict between this statement and the fact the the respective $\alpha_{o x}^{\text {eff }}$ values for the different works are quite similar, because $\alpha_{o x}^{\text {eff }}$ is an extremely "robust" statistic that is dominated by the X-ray loud objects). Avni and Tananbaum (1982) have derived an empirical relation for a dependence of $\alpha_{o x}$ on $L_{o p t}$; our modelindependent, directly observed $\alpha_{o x}$ value for the narrow sample is in excellent agreement with an extrapolation of their prediction, and adds considerable confidence to their claim that this is in fact a physical dependence rather than a subtle mathematical artefact (Chanan 1983). We can also use this argument in a somewhat different context than normally employed, to state that if $\alpha_{o x}$ does not depend on $L_{o p t}$, then, using our derived $\alpha_{o x}$ value and current $\log N / \log S$ counts, we can conclude that $Q S O$ s probably do not dominate the $\mathrm{X}$-ray background radiation; their contribution is $<50 \%$ of the background flux. In short, if one wishes QSOs to make the background, then evolution of $\alpha_{o x}$ must be also accepted.

A significant portion of the background radiation comes from discrete sources unrelated to QSOs, of course; using references cited in Anderson (1985), we estimate 15\% of the flux as due to active galaxies, $5 \%$ to clusters of galaxies, and $5 \%$ to low luminosity active galaxies. If we accept the relationship for $\alpha_{o x}$ functional dependence suggested by Avni and Tananbaum (1982), then the above considerations lead us to conclude that up to $70 \%$ of the background flux can be due to QSOs; this is amazingly close to the fraction unaccounted for by the previously mentioned discrete sources, and the agreement is so 
excellent that it is perhaps fortuitous! Nonetheless we have at least limited, quantitative evidence that QSOs are capable of making the dominant contribution to the background flux.

We close by noting that the combination of the form of the $\alpha_{o x}$ evolution, combined with the shape of the QSO $\log N / \log S$ curve, leads to a specific definition of which QSOs dominate the contributions to the background flux. Although there is of course some contribution from a broad range of objects, this distribution distinctly peaks in the range $\langle z>\sim 1-1.5,19.5<V<20.5$. We thus have the perhaps curious situation that the typical QSO contributing to the background is an intermediate luminosity object in an intermediate redshift annulus of the Universe.

\section{References}

Anderson, S. F. 1985, Ph.D. thesis, University of Washington.

Avni, Y., Soltan, A., Tananbaum, H., Zamorani, G. 1980, Ap. J., 238, 800.

Avni, Y., and Tananbaum, H. 1982, Ap. J. (Letters), 262, L17.

Caillault, J.-P., and Helfand, D. J. 1985, Ap. J., 289, 279.

Chanan, G. A. 1983, Ap. J., 275, 45.

Elvis, M., and Lawrence, A., 1985, in "Astrophysics of Active Galaxies and Quasars", Proc. 7th Santa Cruz Workshop on Astr. and Ap., J. Miller (ed.).

Feigelson, E. D., and Nelson, P. I. 1985, Ap. J., 293, 192.

Giacconi, R., et al. 1979, Ap. J. (Letters), 234, L1.

Gioia, I. M., et al. 1984, Ap. J., 283, 495.

Margon, B., Downes R. A., and Chanan, G. A. 1985, Ap. J. Suppl., 59, 23.

Marshall, H. L., et al. 1984, Ap. J., 283, 50.

Schmitt, J. H. M. M. 1985, A p. J., 293, 178.

Tananbaum, H. et al. 1979, Ap. J. (Letters), 234, L9.

Veron, P. 1983, in "Quasars and Gravitational Lenses", Proc. 24th Liège Int. Ap. Colloq., (Université de Liège), p. 210.

Zamorani, G. et al. 1981, Ap. J., 245, 357.

\section{DISCUSSION}

Schmidt : On the basis of our X-ray observations of bright quasars and the Medium Sensitivity Survey Quasars, Green and I estimate that only $8-13 \%$ of the $2 \mathrm{keV}$ background is contributed by quasars (with $M_{B}<-23$ ).

Margon : First, we should note that the MSS and PG QSOs you discuss both have properties quite different from the sample I discuss here. But, more important, let me stress that our paper states that QSOs can make up to $70 \%$ of the background. This upper limit depends on many uncertain factors, particularly the mean $\mathrm{X}$-ray spectral slope.

Segal : Isn't your finding that there musst be evolution in the $\mathrm{L}_{\mathrm{x}}-\mathrm{L}_{\text {opt }}$ relation if the XRB is to arise in major part from AGNs (\& quasars) restricted to the Friedman cosmology. The chrononetric cosmology, which is non-evolutionary, is consistent with this origin for most of the XRB. 
Margon : It may be that the X-ray astronomy community's insistence on terming possible functional dependences of the $\alpha_{\text {ox }}$ parameter as "evolution" is causing unnecessary semantic confusion. A concise restatement of our finding is that if QSOs are to dominate the X-ray background, it is a necessary (but not sufficient) condition that the ratio of $\mathrm{L}_{\mathrm{x}} / \mathrm{L}$ opt depend on L opt. What cosmological model one wishes to adopt, in addition to this statement, is quite another issue.

Bregman : In addition to reproducing the $\mathrm{X}$-ray background flux, is the spectral shape also reproduced ?

Margon : Some people feel there is a discrepancy between currently measured X-ray spectra of QSOs and that of the background. I am not alarmed yet because the best measurements of these two quantities are in slightly different energy bands. Further, there are just a handful of good QSO $X$-ray spectra available now, and those objects are at lower redshift than the shell that dominates the contribution of QSOs to the background.

Elvis : What level of fluctuation in the IPC deep survey background does your 'thin shell' of quasars predict?

Margon : The shell is not so thin. There is a contribution from a range of objects, with the peak occuring in the $z=1-1.5$ range. For $q_{0}=0$, we are talking about $1 / 3$ of the volume of the Universe ! I am not certain whether the relatively low count rates available in the IPC could detect the background fluctuations due to this large number of objects.

Burbidge : If the X-ray background comes predominantly from QSOS in a restricted range of $z$ does this not put further constraints on the shapes of their spectra?

Margon : It certainly does ! QSOs in the $z=1-1.5$ range will have to have spectra considerably more like the background radiation than had been found for the handful of good QSO spectra currently on hand. But so few good spectra are currently available that I consider the issue essentially unconstrained at the moment.

Cowsik : Would the large no $\sim 10^{6}$ objects needed to explain the isotropy be an argument against QSO's in $\mathrm{z} \sim 1-1.5$ being the sources of the X-ray background.

Margon : There should be no problem satisfying isotropy measurements, because the QSO $\log \mathrm{N} / \log \mathrm{S}$ curve conveniently also peaks near the apparent magnitude range of these $X-$ ray dominant objects : they are very numerous. But a more sensitive imaging $X$-ray telescope than Einstein will resolve most or all of the background sources, if the QSO contribution proves to be near our upper limit. 